\author{
G.J. Pert \\ Department of Applied Physics, University of Hull \\ Hull, HU6 7RX, U.K.
}

\title{
INTRODUCTION
}

In a conventional laser operating in the near ultra-violet, optical or infra-red spectral bands the photon energies, not exceeding $10 e v$, are closely matched to the electronic or molecular energy levels of neutral and weakly ionised atoms. Consequently typical photon energies $(\sim \mathrm{eV})$, and transition lifetimes ( $\sim \mathrm{ns}$ ) closely match the characteristics of fast electrical circuitry feeding a weakly ionised discharge which may be used to pump either directly or indirectly the laser medium.

In a $\mathrm{X}$-ray laser operating at about $10 \AA$, photon energies are about $1 \mathrm{keV}$, and lifetimes about $10^{-14} \mathrm{~s}$ (10fs). In consequence the power required to pump the laser must be expected to increase rapidly as the wavelength decreases. The gain per unit length is given by:

$$
\alpha=\zeta \frac{\grave{\lambda}}{\pi} \frac{\mathrm{A}}{\Delta \nu} \mathrm{g}_{3}\left(\frac{\mathrm{n}_{3}}{\mathrm{~g}_{3}}-\frac{\mathrm{n}_{2}}{\mathrm{~g}_{2}}\right)
$$

where $\zeta$ is the line shape factor, A the spontaneous transition probability, $\lambda$ the wavelength, and $\Delta v$ the width of the line, and $\left(n_{2}, g_{2}\right)$ and $\left(n_{3}, g_{3}\right)$ the population density and statistical weight of the lower and upper laser states respectively. The total power loss per unit area, $p$, of the medium must exceed that emitted by spontaneous decay of the laser transition.

$$
\mathrm{p} \simeq \mathrm{n}_{\mathrm{e}} \ell \cdot \mathrm{h} \nu \mathrm{A}>\frac{8 \pi \mathrm{h}}{\zeta \mathrm{c}^{2}} \nu^{4}(\alpha \ell) \frac{\Delta \nu}{\nu}=\frac{4.10^{21}}{\lambda(\AA)^{4}}(\alpha \ell) \quad\left(\frac{\Delta \lambda}{\lambda}\right) \mathrm{W} / \mathrm{cm}^{2}
$$

where $l$ is the length of the medium. In practical devices we must expect that additional losses will increase the total power demand by as much as an order of magnitude. It must be realised that not only must this power be supplied by an external source, but it must be dissipated by the medium.

In contrast to conventional lasers only two laboratory sources are at present known which can, in principle, deliver the pump power densities $z 10^{15} \mathrm{~W} / \mathrm{cm}^{2}$ required by an $\mathrm{x}$-ray laser namely a subsidiary laser or a particle beam: we shall not discuss a third alternative, a nuclear explosion, as its suitability for non-military applications must be extremely limited. In the less restrictive XUV spectral

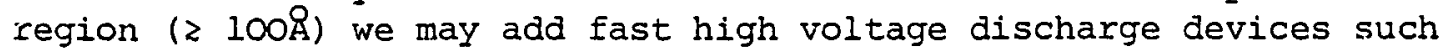
as the superfast pinch.

The problem is further complicated by the absence of simple transmitting or reflecting media at $x$-ray wavelengths so that the constraints cannot be relaxed by allowing the laser signal to build up in a resonant cavity, or distributed feedback system. At XUV wavelengths metals at grazing incidence must be used for mirrors in order to obtain high reflectivity, and at X-ray wavelengths crystal reflectors, but again at non-normal incidences. Under such conditions 
the construction of a cavity is extremely complex. The recent development of multi-layer reflectors in this wavelength range may eventually solve this difficulty but for the present their reflectivity is low (Haelbich et al 1980; Barbee and Keith 1979). The absence of a cavity implies that the laser must operate in a weakly coherent travelling wave, or amplified spontaneous emission mode. This form of operation in which the spontaneous fluorescence is amplified by stimulated emission down a rod shaped medium requires a gain-length product $(\alpha l) \sim 20$ (i.e. an overall amplification $e^{\alpha l}$ of about $100 d b$ ). Such a device produces a beam whose divergence will be limited by either diffraction or geometry to approximately the larger of two values $\lambda / d$ and $d / l$, where $d$ is the rod diameter and $l$ its length. If. these are matched the divergence is minimised, and furthermore the beam will be coherent across its wavefront. Thus ideally the diameter should have the optimum value

$$
\mathrm{d} \simeq \sqrt{\lambda \ell}
$$

If the diameter is less than this value diffraction losses will reduce the effective gain length to a value $\ell^{1} \simeq d^{2} / \lambda<\ell$.

Since the total power $P \simeq p d^{2}$ it is clear that if the total pump power is to be kept within reasonable limits (say 100Tw) then $d s$ $10^{-3} \lambda(\AA)^{2} \mathrm{~cm}$, and therefore $\ell \leqslant 10^{-6} \lambda(\AA)^{3} \mathrm{~cm}$, assuming $\Delta \lambda / \lambda \cong 10^{-4}$. Hence we may estimate that the minimum inversion density $\Delta n \simeq 6 \times 10^{21}$ $/ \lambda(\AA) 4 / c c$ assuming the oscillator strength of the lasing transition at unity.

It is clear from the preceeding discussion that the principal limitations on constructing an $X$-ray laser is the high pump power required. In principle we may satisfy this condition either directly in which case the external pump must itself satisfy the power condition, or indirectly when the system is pumped to a relatively long lived reservoir state, which may be slowly filled, to be transferred into the upper laser state by some appropriate trigger. The latter approach relaxes the condition on the external pump by using the reservoir energy, but is clearly limited to short pulse action only.

\section{RECOMBINATION LASERS}

One of the most promising approaches to a laboratory XUV laser at present seems to be the recombination laser. Population inversion generated in this way is a familiar feature in many laser produced plasmas, and significant gain has been measured. The inversion is achieved during the cascade through the excited states of highly charged ions following recombination into a high lying state. Two basic approaches can be envisaged, requiring different forms of the ion term scheme. If the principal de-excitation process in the cascade is via electron collisions, the inversion forms between a pair of well separated levels within a group of closely spaced ones. In contrast with radiative decay the inversion generally forms between a pair of levels, the lower of which is well separated from its next lower state, for example, the $2 p$ state of hydrogen. The first approach has been successfully applied by workers at Bell Labs in the visible region of the spectrum (Silfvast and Wood, 1980), whereas the latter has been used in the XUV with emphasis on hydrogenlike ions (Pert, 1976), it being relatively easy to produce a high fractional population of the parent ion by stripping the medium. In 
order to achieve significantly large gain within the recombination cascade, it is necessary to first ionise the medium, and then rapidly cool it so that the dominant recombination is into high lying states rather than directly into the ground state. This, the basic problem, may be accomplished in a number of ways of which adiabatic cooling by expansion (Pert, 1976) and radiative heat loss (Suckewer and Fishman, 1980) are the most promising.

There have, within the past few years, been several reports of gain either measured directly or indirectly from expansion cooled recombination systems. Using slab targets irradiated with Nd:glass laser pulses of lOns duration Elton and co-workers at N.R.L. (1982) have measured a gain of $2 \% / \mathrm{cm}$ at $520 \AA$ on the hydrogen-like carbon lines CVI, $P_{\alpha}$. Jaeglé and associates at orsay (198I) with variable length line focussed Na;glass laser pulses also of ns duration on aluminium targets have observed gain of the lithium-like aluminium line Al XI, $3 \mathrm{~d}-5 \mathrm{f}$ at $105.7 \AA$ with values $\sim 2 / \mathrm{cm}$ over lengths of plasma up to $1 \mathrm{~cm}$. The largest gain has been measured in hydrogen-like carbon $\mathrm{CVI}, \mathrm{H}_{\alpha}$ at $182 \AA$ with values of $\sim 25 / \mathrm{cm}$ over $2 \mathrm{~mm}$ length by the Hull University group (Jacoby et al, 1982).

The plasma must first be rapidly heated, and then cooled over timescales of the order of the characteristic ionisation time: for CVI ions at densities $\sim 10^{21}$ ions/cc this implies times of order 100ps. These times are characteristic of the duration of mode-locked pulses from a Nd:glass laser, and of the expansion of plasma of appropriate temperature $\sim 10^{-3} \mathrm{~cm}$ in size. In practical terms these parameters closely match those obtained by heating carbon fibres of a few microns diameter with an appropriate laser pulse, a conclusion confirmed by detailed computer modelling (Pert, 1976). In this system the inversion is achieved at sufficiently high density ( $10^{19}$ ions/cc) to give an inversion density $\sim 1015 / \mathrm{cc}$ with gain coefficients $\sim 10 / \mathrm{cm}$, sufficient to measure in a laboratory experiment.

\section{CARBON FIBRE LASER SCHEME}

The use of thin carbon fibre irradiated by cylindrically focussed Nd:glass laser radiation, and its harmonic as a working medium for laser action in the XUV has been investigated both experimentally and theoretically. In this system rapid cooling by adiabatic expansion of the fully stripped carbon plasma induces population inversion between the $n=3$ and $n=2$ levels of the hydrogenic like carbon ion CVI during the recombination cascade. In general the gain in such a system is small, limited by the optical trapping of the lower laser level $n=2$. However, if the scheme is to have an application with current technology amplified spontaneous emission of travelling wave mode of operation is necessary, requiring a relatively high gain. This condition can only be achieved if the resonance line can be made optically thin at a reasonably high density.

In the carbon fibre system this handicap is mitigated by two effects. Firstly the carbon is initially fully stripped, and oniy relatively weakly ( $\sim 10 \%$ ) recombined at gain onset to reduce the Lyman $\alpha$ ground state population. Secondly the effective plasma width is kept small. This latter condition is achieved both directly by using fibres of small initial diameter, but more importantly due to the approximately linear dependence of the radial velocity, and the motional Doppler effect, which ensures that only a limited width of 
the plasma is in resonance at line centre.

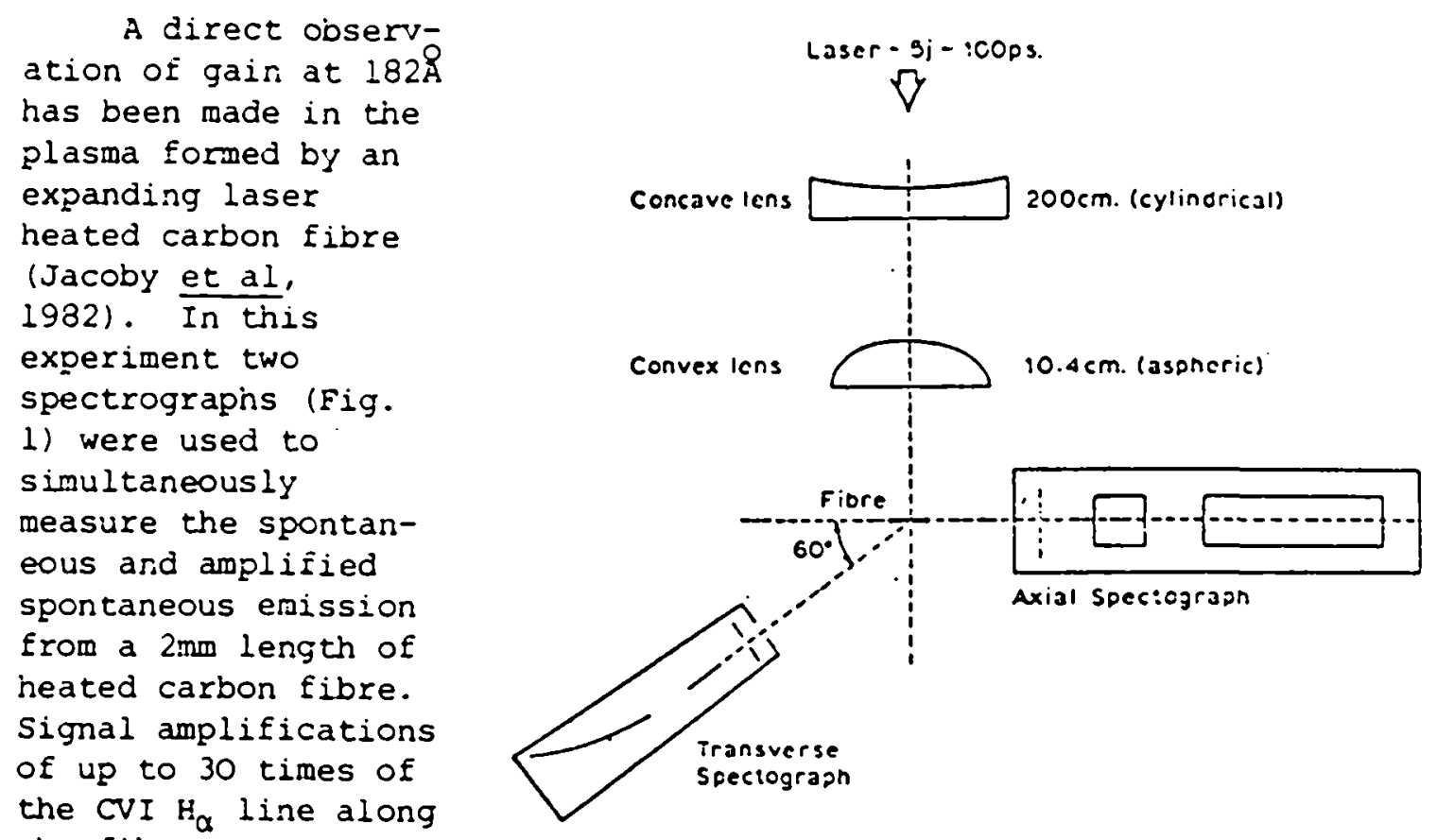

the fibres were measured and the inferred gain/length parameters are shown in Fig. 2. The strong variation with laser energy shown is in good agreement with computer modelling of the interaction. The need for extreme care in cross calibrating the spectrographs in any experiment of this type should be noted.

It was found that the measured plasma parameters were only weakly dependent on the fibre diameter, provided it was not too large ( $\leqslant 6 \mu \mathrm{m})$. This was inferred to be due to an incomplete burn of the fibre, the actual mass participating in the gain generation being strongly dependent on the absorbed laser energy. This

Fig. I Diagram of the apparatus used in the University of Hull experiments to measure gain at $182 \AA$ from laser heated carbon fibres.

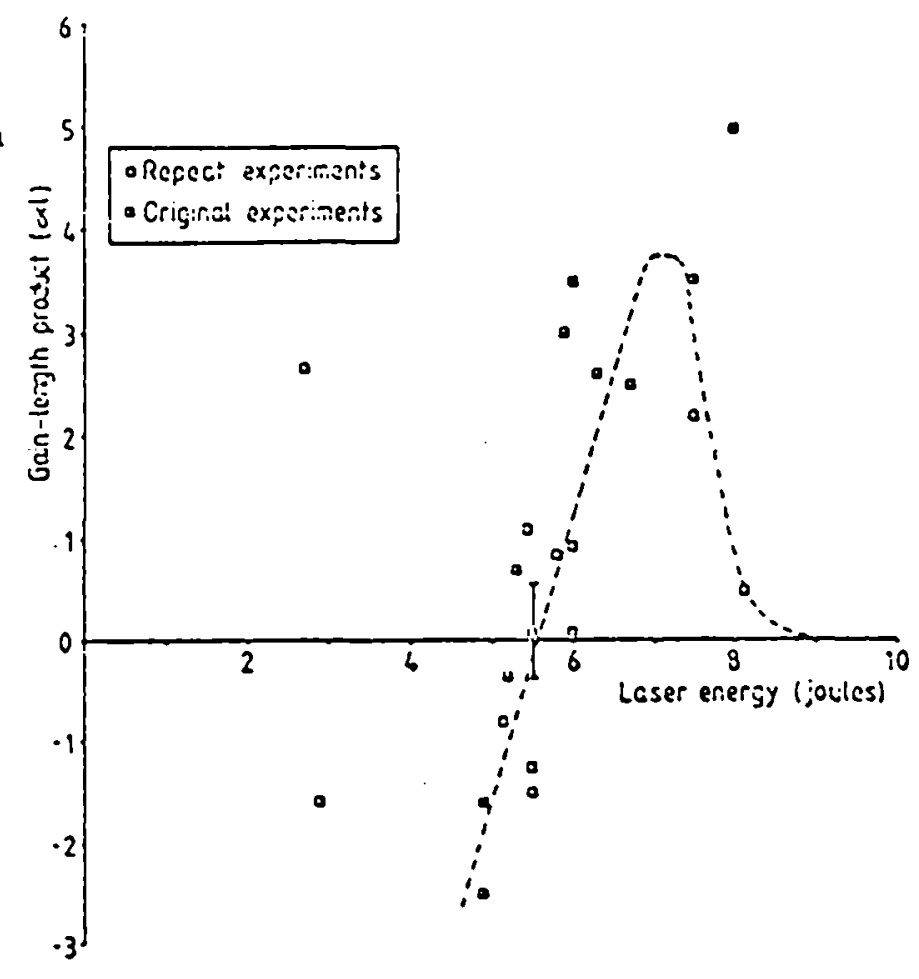

Fig. 2 Measured gain as a function of input laser energy in the low mass regime. Closed points refer to old data of Jacoby et al (1982) and open ones to repeat experiments. For comparison laser energy of $8 j$ is approximately equivalent to absorbed density of $2 \mathrm{tj} / \mathrm{cm}$ in Fig. 3. 
result has been confirmed in subsequent experiments, and the mass/energI relationship

deduced for a number of pump laser configurations (Pert et al 1984) Fig. 3. Since the condition for peak gain can also be expressed in terms of an energy/mass relation, this offers the important prospect of matching the burn to the gain by the use of appropriate pump laser parameters. Since the curve overlap can be large this has two attractive features. Firstly, by operating at low energy/low mass the gain can be maximised as in the experiments of Jacoby et al (1982). Secondly, fluctuations in the absorbed energy along the line of the fibre due to shot-to-shot variation, and to imperfect lens design can be accommodated whilst still maintaining high gain by shifts along the gain-optimised mass/energy curve.

The importance of operation in the low mass/low energy regime and of burn/gain matching has been clearly demonstrated in high energy experiments at the C.L.F. (Pert et al, 1984) in which only relatively weak gain was observed, but which confirmed the role of incomplete heating.

Two distinct measurements of the effective mass were carried out. Simple ion probe measurements were made routinely. In addition interferograms allowed a direct observation of the density profile of the plasma which can be integrated to give a check on the ion probe results. Excellent agreement between the data validated the ion probe method. These results are compared with the computational predictions of 1 and 2 dimensional codes in fig. 4, with good overal.1 agreement.

The interferograms clearly show the presence of the cold dense core, whose radius is much less than that of the expanding plasma. Density profiles measured from two shots with very similar effective mass and absorbed energy at different times are compared with the calculated distribution predicted by the 1 dimensional code in fig. 5 . 
When allowance is made for the uncertainty of the timing zero of the laser pulse, the agreement is most satisfactory.

$\quad$ One of the
attractive
features of the
recombination
laser in
hydrogen-like
ions is that
the scaling
along the iso-
electronic
sequence is
relatively well-
known (Pert 1976)
and thus systems
can be extrapo-
lated towards
x-ray wave-
lengths with
some degree of
confidence. The
analysis given
in Pert (1976)
indicates similar
behaviour can be
expected for
ions of charge
number $\mathrm{z}$, if
the tenperature

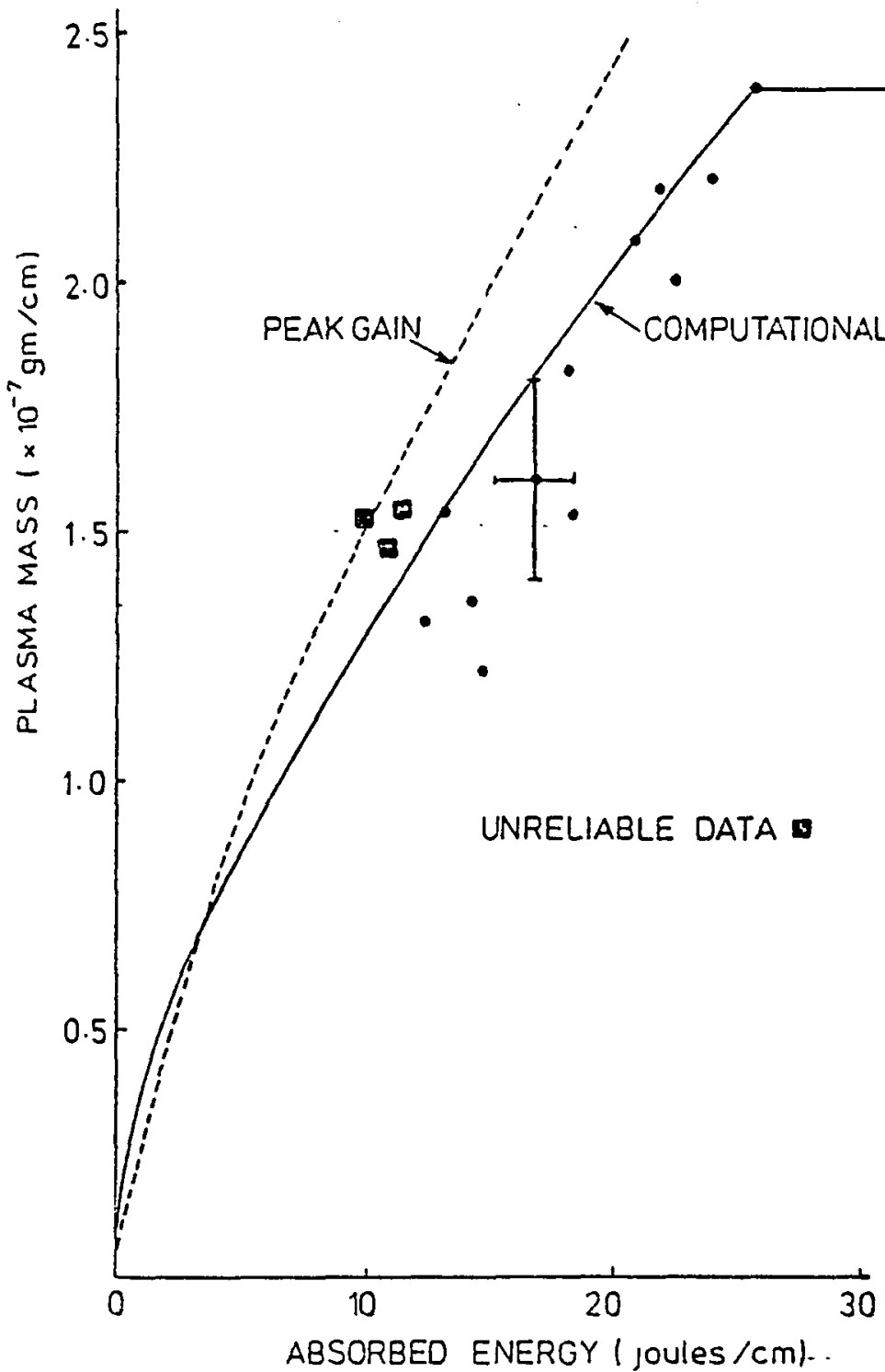

Fig. 4 Mass energy/plot for the high mass interferometry experiment; the data is presented as in fig. 3.

the temperature
is scaled as $z^{2}$, the diameter as $z^{-3}$, the ion density as $z^{6}$. The resultant gain scales as $z^{7.5}$. Since the lasing wavelength scales as $z^{-2}$, some estimates can be made of the necessary conditions for short wavelength. If this is done a laser operating with aluminium at about $39 \AA$ appears to be feasible using thin foils irradiated with pulses of about lops duration (Davé and Pert 1984). For shorter wavelengths the scaling is at present more speculative.

\section{ACKNOWLEDGEMENTS}

It is a pleasure to acknowledge the contributions made by many colleagues to this project: in particular Drs. Jacoby, Shorrock and Tallents at University of Hull, Drs. Lamb and Lewis, Mr. Corbett and Miss Mahoney at Queen's University, Belfast, and Drs. Eason, Hooker, Key and Pivizhong at the Rutherford Laboratory. The programme is supported by S.E.R.C. 


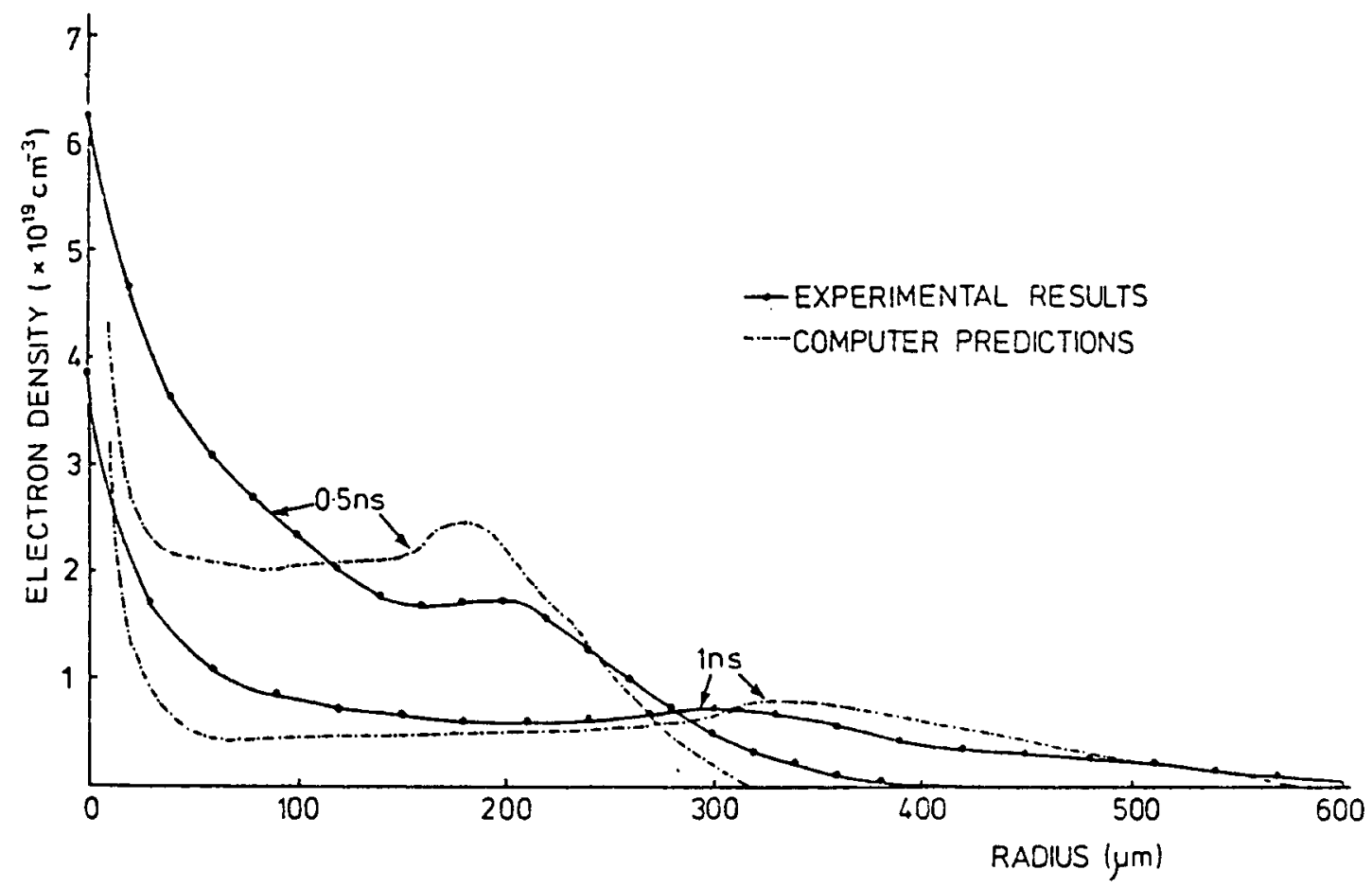

Fig. 5 Comparison of experimental and computational electron density profiles at two times during the expansion of an incompletely burnt fibre with effective mass $1.5 \times 10^{-7} \mathrm{gm} / \mathrm{cm}$ and absorbed energy $13 \mathrm{j} / \mathrm{cm}$.

\section{REFERENCES}

Haelbich R.Pl, Segmuller A. and Spiller E. 1980, Appl. Phys. Lett. 34,6 .

Barbee T.W. Jr. and Keith D.L. 1979 in Lithography/Microscopy Beamshine Workshop, ed. C.R. Dannemiller. SSRL Report No. 79/02, 185. Silfvast W.T. and wood O.R. Jr. 1980, J. de Physique, 41, C9, 439. Pert G.J., 1976, J. Phys. B, 9, 3301.

Suckewer S. and Fishman H. 1980, J. Appl. Phys., 51, 1922.

Elton R.C., Seely J.F. and Dixon R.H., 1982, Paper presented at lst Topical Meeting on Laser Techniques in the Extreme Ultraviolet, Boulder, USA.

Jamelot G., Jaegle P., Carillon A., Bideau A., Moller C., Guennou H. and Sureau A., 1981, in Proc. Int. Conf. on Lasers ' 81 , New Orleans, December 1981, ed. C.B. Collins (S.T.S. Press, McLean Va.) 178.

Jacoby D., Pert G.J., Shorrock I.D. and Tallents G.J., 1982, J. Phys. $B, 15,3557$.

Pert G.J., Shorrock L.D., Tallents G.J., Corbett R., Lamb M.J., Lewis C.L.S., Mahoney E., Eason R.B., Hooker C. and Key M.H., 1984, Paper presented at IInd Topical Meeting on Laser Techniques in the Extreme Ultraviolet, Boulder, USA, and Central Laser Facility Annual Report, RAL 84.049, A4.2.

Davé A.K. and Pert G.J., J. Phys. B: in press. 